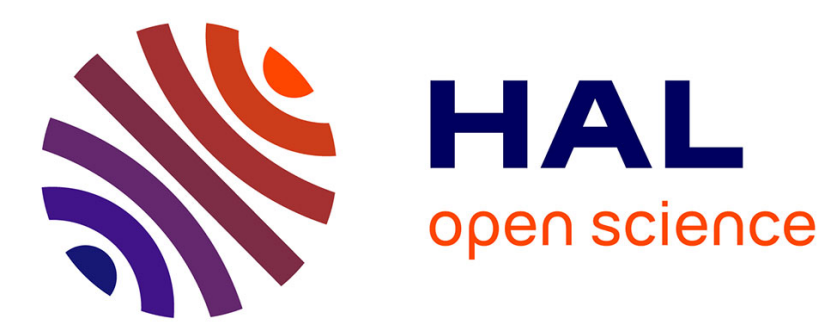

\title{
Positron reactions with SF6 in hexane studied by measurements of the Doppler broadening of the annihilation line
}

O. Mogensen, T. Hirade

\section{- To cite this version:}

O. Mogensen, T. Hirade. Positron reactions with SF6 in hexane studied by measurements of the Doppler broadening of the annihilation line. Journal de Physique IV Proceedings, 1993, 03 (C4), pp.C4-17-C4-27. 10.1051/jp4:1993402 . jpa-00251445

\section{HAL Id: jpa-00251445 \\ https://hal.science/jpa-00251445}

Submitted on 1 Jan 1993

HAL is a multi-disciplinary open access archive for the deposit and dissemination of scientific research documents, whether they are published or not. The documents may come from teaching and research institutions in France or abroad, or from public or private research centers.
L'archive ouverte pluridisciplinaire HAL, est destinée au dépôt et à la diffusion de documents scientifiques de niveau recherche, publiés ou non, émanant des établissements d'enseignement et de recherche français ou étrangers, des laboratoires publics ou privés. 


\title{
Positron reactions with $\mathrm{SF}_{6}$ in hexane studied by measurements of the Doppler broadening of the annihilation line
}

\author{
O.E. MOGENSEN and T. HIRADE
}

Chemistry Department, Japan Atomic Energy Research Institute, Tokai-mura, Ibaraki-ken 319-11, Japan

non-refereed paper

\begin{abstract}
The Doppler broadening (DB) of annihilation radiation spectra were measured for solutions of $\mathrm{SF}_{6}$ in hexane at $\mathrm{SF}_{6}$ concentrations up to $1 \mathrm{M}$. By use of a new program GAUSSFIT the DB-spectra were analyzed in terms of a sum of the resolution-convoluted angular correlation spectrum for hexane and one or more extra, gaussians. Positron annihilation with electrons bound in fluorine gives an unusually broad DB-component. This effect was used to show that the positron reacts with $\mathrm{SF}_{6}$ in hexane with a rate constant of $2.3 \times 10^{10} \mathrm{M}^{-1} \mathrm{~s}^{-1}$. The result agrees with previous predictions based on positronium yield results and the spur model of Ps formation.
\end{abstract}

\section{Introduction}

Positronium (Ps) the bound state of an electron and a positron is formed in many molecular liquids and solids. The yield of Ps can be determined from the intensity of the long-lived orthoPs pick-off component of the positron lifetime (LT) spectra and/or from the intensity of the narrowest component in the angular correlation (AC) spectra. The changes in the Ps yields on addition of many solutes to liquids have attracted much attention during the last decades [1-4].

About ten years ago experimental facts indicated that the spur model of Ps formation could not explain the Ps yield results for solutions of electron scavengers trapping electrons in weakly bound, stable anion states and non-polar liquids in terms of only the electron acceptor properties of the scavengers. Typically, the studied scavengers had electron affinities of $0.5-1.0 \mathrm{eV}$ in vacuum. For example, some scavengers, such as $\mathrm{C}_{6} \mathrm{~F}_{6}[5]$ and naphthalene [5], increased, while other scavengers, e.g., $\mathrm{SF}_{6}[6], \mathrm{CS}_{2}[7], \mathrm{C}_{6} \mathrm{~F}_{14}$ [8], and $\mathrm{CH}_{3} \mathrm{Br}$ [9] decreased, the Ps yield when added to hexane at low concentration. It was then realized that the positron acceptor properties of the added molecules played an important role in the Ps formation processes, too [9]. The measured Ps yield could only be explained, if it was assumed that addition of a solute reduced the Ps yield only if both the electron and the positron were trapped on a solute forming weakly bound anions on electron addition. The mobility and diffusion constant of the trapped particles are much smaller than those of the free particles, which implies that the positron can annihilate before Ps is formed in many cases where Ps formation would have occurred in the pure solvents. If only one of the particles is trapped on the solute the other particle can pick off the trapped particle to form Ps in time before the positron annihilates. For example, the positron is not 
trapped on $\mathrm{C}_{6} \mathrm{~F}_{6}$ and naphthalene in hexane. Hence, as the positron is fast moving in hexane it can pick of the electron trapped on $\mathrm{C}_{6} \mathrm{~F}_{6}$ and naphthalene before the slowly moving positive ion can do it, and the Ps yield is, therefore, increased if these molecules are added to hexane. This is the well-known anti-inhibition effect in the Ps formation. The measured Ps yields could be well explained by the intproved spur model [9].

Of course, it is important to show directly that the positron is actually trapped on the electron acceptor molecules giving Ps inhibition and not on those resulting in Ps anti-inhibition, apart from the effects on the Ps formation. However, it is very difficult to measure directly the trapping of the positron on molecules, in general. The lifetimes of the positron is almost identical for most positron states in liquids and solutions. The angular correlation and Doppler broadened (DB) annihilation lines are "fingerprints" of the positron-electron states on annihilation. Hence, these states can be identified in a solution if the positron states found in the solution differ enough to permit a clear separation of the AC- or DB-components. In particular, positron annihilation with electrons bound in fluorine atoms in molecules results in an unusually broad $\mathrm{AC}$ - or DB-component [4,10]. Consequently, the relative percentage of positrons forming bound states with perfluorinated molecules can fairly easily be determined by use of AC-and DBmeasurements.

The AC-results for $\mathrm{C}_{6} \mathrm{~F}_{14}$ mixtures with hexane and isooctane showed clearly that the broad components of the AC-spectra were strongly broadened on the addition of a small concentration of $\mathrm{C}_{6} \mathrm{~F}_{14}$ [11], while a similar addition of $\mathrm{C}_{6} \mathrm{~F}_{6}$ to hexane did not cause a broadening of the broad AC-component [10]. Hence, the positron reacts with $\mathrm{C}_{6} \mathrm{~F}_{14}$ in hexane and isooctane to form a bound state, where the positron annihilates preferentially with the electrons in the fluorine atoms, while it does not react with $\mathrm{C}_{6} \mathrm{~F}_{6}$ in hexane. This was expected from the Ps yield results. Of course, it is useful to find other molecules than $\mathrm{C}_{6} \mathrm{~F}_{14}$ which react with the positron.

Hence, we decided to investigate solutions of $\mathrm{SF}_{6}$ in hexane to look for the expected broadening of the broad DB-component, for the following reasons. $\mathrm{SF}_{6}$ is a standard electron scavenger in radiation chemistry [12], and it forms a stable anion by a reaction with the excess electron in hydrocarbons. It is also structurally different from perfluorinated hydrocarbons. Furthermore, it inhibits Ps formation in many hydrocarbons [5,6] at low concentration. However, it can only inhibit the Ps yield to a certain minimum (e.g., to about $16 \%$ in hexane), which is typical for scavengers forming stable anions. Another reason was that only a DB-apparatus was available to us. Although the $\mathrm{AC}$ - and $\mathrm{DB}$-spectra are determined by identical properties of the annihilating positron-electron system, AC-measurements have a much better resolution than DBmeasurements. However, $\mathrm{SF}_{6}$ solutions at high enough concentration in hydrocarbons have a too large pressure of $\mathrm{SF}_{6}$ above the solution to permit well-defined $\mathrm{AC}$ measurements. Hence, it is necessary to used $\mathrm{DB}$-measurements to study the positron $\mathrm{SF}_{6}$ reaction in these solutions.

We performed also the DB-measurements because a new computer program, GAUSSFIT, was available to us [13]. It has been developed partly to analyze DB-spectra in terms of previously obtained AC-results. We wished to test the program in the analyses of the DB-spectra for $\mathrm{SF}_{6}$ hexane solutions. This type of detailed analyses of DB-data has not been performed earlier.

Lifetime spectra were also obtained for all the solutions. They will mainly be discussed in a separate paper.

The experimental conditions are discussed in sect. 2. In sect. 3 the computer analyses and the obtained results are described in some detail. A discussion of the results is given in sect. 4, followed by a conclusion in sect. 5 . 


\section{Experimental}

The Doppler broadening (DB) of the annihilation line was measured by use of a high-purity germanium detector. The full width at half maximum (fwhm) of the detector resolution was about $1.33 \mathrm{keV}$ (see sect. 3). It was determined as the resolution of the ${ }^{85} \mathrm{Sr} 513.998 \mathrm{keV}$ line. The energy width per channel obtained from the positions of the ${ }^{85} \mathrm{Sr}$ and the ${ }^{137} \mathrm{Cs} 661.63 \mathrm{keV}$ lines was about $85 \mathrm{eV}$. The positron source was roughly $1.85 \times 10^{5} \mathrm{~Bq}(5 \mu \mathrm{Ci}){ }^{22} \mathrm{NaCl}$ between two 1.1 $\mathrm{mg} / \mathrm{cm}^{2}$ Kapton foils. As a spectrum stabilizer was not included in the set-up, and since the temperature of the room was not well stabilized, we determined the resolution just before and just after the measurements of each DB-spectra. The use of the resolution spectra is discussed in sect. 3.

The LT-spectra for all the $\mathrm{SF}_{6}$ hexane solution were measured by use of a standard fast-fast LTapparatus, with a resolution fwhm of about 250 ps. We used the same source as in the DB measurements. The details of the high-quality LT-measurements and data analyses were discussed in detail in [14].

The AC-spectra were measured by use of a standard linear-slit AC-apparatus with a resolution of about fwhm $=1.15 \mathrm{mrad}$, of which $0.96 \mathrm{mrad}$ was determined by the slit widths $[10,15]$.

All the samples used for the DB-, LT-, and AC- measurements were degassed by means of the well-known freeze-thaw technique. After degassing the $\mathrm{SF}_{6}$ solutions were prepared on a vacuum line by use of the standard pv-technique.

\section{Analyses and Results}

The analyses of the DB-spectra were fairly complicated and, apparently, the first of their kind. Consequently, a detailed discussion is appropriate.

The DB-spectra were analyzed by use of the fortran program, GAUSSFIT. GAUSSFIT fits a spectrum, given by a table of measured numbers, $y_{i}$, by a sum of gaussians plus a constant. All the $x$ coordinates, $x_{i}$, are assumed to be separated by the same constant value. Hence the input spectrum is roughly similar to that of the well-known POSITRONFIT and RESOLUTION $[16,17]$. A normalized gaussian is given by:

$$
\mathrm{G}_{\mathrm{i}}(\mathrm{x})=\left((2 \pi)^{-1 / 2} / \sigma_{\mathrm{i}}\right) \exp \left\{-\left(\mathrm{x}-\delta_{\mathrm{i}}\right)^{2} /\left(2 \sigma_{\mathrm{i}}^{2}\right)\right\}
$$

The fitting sum is

$$
\mathrm{F}(\mathrm{x})=\sum_{i} \mathrm{~L}_{\mathrm{i}} \mathrm{G}_{\mathrm{i}}(\mathrm{x})+\mathrm{c}
$$

The fitting parameters are $\mathrm{I}_{\mathrm{i}}, \sigma_{\mathrm{i}}$, and $\delta_{\mathrm{i}}$ for each gaussian, and the constant $\mathrm{c}$. All parameters can be fixed to a given value in the fitting, and linear combinations of the intensities, $I_{i}$, can be fixed to be a constant. Hence, GAUSSFIT is fairly similar to the programs PAACFIT and ACARFIT of the PATFIT package, except that the positions of the gaussians are fitting parameters, too.

At first, GAUSSFIT was tested on many artificially calculated spectra. Later it was used to fit about 150 X-ray fluorescence (XRF) spectra. Actually, P. Kirkegaard used $2-3$ weeks to make the first version of the program, and about ten errors were detected during the tests lasting about five months. In the last part of the XRF analyses and in the series of analyses discussed here thousands of fits were performed and no errors detected. 
Before the GAUSSFIT analysis the measured DB-spectra were treated by the fortran program, DOPPLER, in the following way. First, the best possible "symmetry" $x$-coordinate, AVX, of the somewhat asymmetric main peak (511 numbers) was determined. AVX was used in the source component and background subtractions and the calculation of the peak parameters. Next, a spectrum due to annihilation in the Kapton-covered source was subtracted. This source spectrum was assumed to be fairly well described by the spectrum for a thick Kapton sample having an area of $10 \%$ of the area of the measured spectrum. The "symmetry" axis of the Kapton spectrum was known, and linear interpolation between the points of the source spectrum was used in the subtraction. The background was then subtracted as follows. The roughly constant backgrounds just above (10 keV $<E<19 \mathrm{keV}), B_{a}$, and just below $(-19 \mathrm{keV}<E<-10 \mathrm{keV}), B_{b}$, the main peak were determined. At a given point, $j$, of the main peak, the area of the main peak above that point, $A_{j}$, was determined. A background, $B_{g}=B_{a}-\left(B_{b}-B_{a}\right) A_{j} / A_{p}$, was subtracted. $A_{p}$ is the total area of the main peak. Both $A_{j}$ and $A_{p}$ were calculated by use of simple summation of the measured counts. Four spectrum parameters were then calculated. For example, the H-parameter defined as the ratio of the areas between, e.g. $\pm 0.1277 \mathrm{keV}$ and $\pm 3.8325 \mathrm{keV}$, and the $\mathrm{W}$ parameter given by the ratio of the areas at the wings of the main spectrum and the area \pm 3.8325 $\mathrm{keV}$, were determined. All the used areas were calculated by means of linear interpolation between measured numbers and limits given as distances from the symmetry axis. At last, the main peak normalized to a standard area was output in a format ready for GAUSSFIT analysis. This is the main treatment of DB-spectra used for many years at Risø, Denmark.

The GAUSSFIT analysis of a pure hexane spectrum was performed as follows. We calculated the DB-spectrum expected from the three gaussian fit of the AC-spectrum for pure hexane, given in table 1. This fitting curve fitted the AC-spectrum well. To deconvolute the AC-curve for the resolution of fwhm $=1.15 \mathrm{mrad}(0.2938 \mathrm{keV})$ and to convolute the resulting AC-curve with the resolution of the DB-apparatus we used the following expression [18]:

$$
\begin{aligned}
& \left(2 \pi \sigma_{1} \sigma_{2}\right)^{-1} \int_{-\infty}^{\infty}\left[\exp \left\{-\left(x^{\prime}-x^{\prime \prime}\right)^{2} /\left(2 \sigma_{1}^{2}\right)-\left(x^{\prime \prime}-x\right)^{2} /\left(2 \sigma_{2}^{2}\right)\right\}\right] d x^{\prime \prime} \\
& =\left(2 \pi \sigma_{t}^{2}\right)^{-1 / 2} \exp \left\{-\left(x^{\prime}-x\right)^{2} /\left(2 \sigma_{\mathrm{t}}^{2}\right)\right\}
\end{aligned}
$$

where $\sigma_{t}^{2}=\left(\sigma_{1}^{2}+\sigma_{2}^{2}\right)$.

Hence, a good approximation of the "true" AC-curve or DB-curve for hexane is a sum of three gaussians with the same intensities as the measured AC-curve and fwhm's given by

fwhm $m_{t, i}=\left(\mathrm{fwhm}_{\mathrm{i}}^{2}-\mathrm{fwhm}_{\mathrm{r}}^{2}\right)^{1 / 2}$, where $\mathrm{fwhm}_{\mathrm{r}}=1.15 \mathrm{mrad}$ is the AC-resolution fwhm. By the "true" curve we mean the curve that one would measure, if the resolution were infinitely high.

We know that $\sigma_{\mathrm{i}}=0.42466 \times \mathrm{fwhm}_{\mathrm{i}}$. Additionally, the AC-angle, $\Theta$, is given by the measured DBenergy, E, by:

$$
\Theta=\left(2 / m c^{2}\right)\left(E-m c^{2}+E_{b} / 2\right)
$$

where $2 /\left(\mathrm{mc}^{2}\right)=3.91390 \mathrm{mrad} / \mathrm{keV}, \mathrm{mc}^{2}$ is the rest energy of the positron and electron, and $\mathrm{E}_{\mathrm{b}}$ is the binding energy of the electron-positron pair in the sample. In the case of an anisotropic sample the DB-detector must be placed along the z-axis of the AC apparatus, e.g., above the sample if the slits of the AC-setup are horizontal, for (4) to be correct. As $E_{b}$ is only a few eV, we can disregard $E_{b}$ here. Hence, the true DB-curve is well approximated by a sum of three gaussians:

$$
\mathrm{G}(\mathrm{E})=\sum_{j} \mathrm{~T}_{\mathrm{j}} \mathrm{G}_{\mathrm{j}}(\mathrm{E})
$$

for which we know the parameters of the three gaussians. The spectrum and the associated 
Table 1. Typical examples of the fitting of some spectra. I, fwhm, and pos: are the relative intensity, full width at half maximum, and position of the gaussians in a fit of a sum of gaussians to the spectra, respectively.

\begin{tabular}{|c|c|c|c|c|c|c|c|c|c|}
\hline Spectrum & $\begin{array}{l}I \\
(\%)\end{array}$ & $\begin{array}{l}\text { fwhm } \\
(\mathrm{keV})\end{array}$ & $\begin{array}{l}\text { pos. } \\
\text { (keV) }\end{array}$ & $\begin{array}{c}I \\
(\%)\end{array}$ & $\begin{array}{l}\text { fwhm } \\
(\mathrm{keV})\end{array}$ & $\begin{array}{l}\text { pos. } \\
(\mathrm{keV})\end{array}$ & $\begin{array}{c}I \\
(\%)\end{array}$ & $\begin{array}{l}\text { fwhm } \\
(\mathrm{keV})\end{array}$ & $\begin{array}{l}\text { pos. } \\
\text { (keV) }\end{array}$ \\
\hline AC hex. & 16.66 & 0.649 & 0.0 & 80.88 & 2.190 & 0.0 & 2.45 & 5.197 & 0.0 \\
\hline AC hex. dec. & .16 .66 & 0.577 & 0.0 & 80.88 & 2.169 & 0.0 & 2.45 & 5.189 & 0.0 \\
\hline $\mathrm{AC} \mathrm{SF}_{6}-44 \mathrm{C}$ & 9.632 & 0.605 & 0.0 & 47.922 & 2.444 & 0.0 & 42.447 & 3.714 & 0.0 \\
\hline $\mathrm{AC} \mathrm{SF}_{6}-88 \mathrm{C}$ & -2.174 & 1.535 & 0.0 & 93.258 & 3.017 & 0.0 & 8.916 & 19.258 & 0.0 \\
\hline \multirow{3}{*}{ DB Sr-85 } & 99.38 & 1.310 & 0.0 & & & & & & \\
\hline & 0.234 & 1.500 & 1.453 & & & & & & \\
\hline & 0.385 & 4.417 & 3.968 & & & & & & \\
\hline \multirow{4}{*}{ DB hex. calc. } & 16.557 & 1.432 & 0.0 & 80.379 & 2.535 & 0.0 & 2.436 & 5.351 & 0.0 \\
\hline & 0.039 & 1.608 & 1.453 & 0.189 & 2.638 & 1.453 & 0.006 & 5.401 & 1.453 \\
\hline & 0.064 & 4.454 & 3.968 & 0.311 & 4.921 & 3.968 & 0.009 & 6.814 & 3.968 \\
\hline & & $=1-3)$ & & & $i=4-6$ & & & $1-2,1$ & \\
\hline
\end{tabular}

resolution spectra were analyzed in terms of three gaussians plus a constant as given by (2). We then know the DB resolution curve given by :

$$
\mathrm{F}(\mathrm{E})=\sum_{i} \mathrm{I}_{\mathrm{i}} \mathrm{G}_{\mathrm{i}}\left(\mathrm{E}-\delta_{\mathrm{i}}\right)
$$

Hence, by use of (3) we can calculate the expected, measured DB-curve given by a sum of nine gaussians by folding (5) and (6):

$$
\mathrm{f}(\mathrm{E})=\int_{-\infty}^{\infty} \mathrm{F}\left(\mathrm{E}^{\prime}-\mathrm{E}\right) \mathrm{G}\left(\mathrm{E}^{\prime}\right) \mathrm{dE^{ \prime }}=\sum_{j} \sum_{i} \mathrm{I}_{\mathrm{i}} \mathrm{T}_{\mathrm{j}} \mathrm{G}_{\mathrm{ij}}\left(E-\delta_{\mathrm{i}}\right)
$$

where $\mathrm{G}_{\mathrm{ij}}$ is given by (1) with $\sigma_{\mathrm{ij}}=\left(\sigma_{\mathrm{i}}^{2}+\sigma_{\mathrm{j}}^{2}\right)^{1 / 2}$. Such DB-curve is given by the nine gaussians marked DB hex. calc. in table 1 . It is obtained by folding the curves marked AC hex.dec. and the resolution curve, DB Sr-85, in table 1.

A curve of this shape was then fitted to the measured DB-spectrum for hexane, which had been treated by a DOPPLER analysis. The fwhm $\mathrm{m}_{\mathrm{i}}, \mathrm{i}=1, \ldots, 9$, were fixed to the values given in table 1 (e.g, fwhm $_{1}=1.432 \mathrm{keV}$ ). Eight linear combinations of the intensities, $I_{1}, \quad A_{i} \times I_{4}-A_{4} \times I_{1}=0$, $i=1, \ldots, 3,5, \ldots, 9$, were used to fix the ratios of the intensities equal to those of the intensities, $\mathrm{A}_{\mathrm{i}}$, given in table 1 (e.g., $16.557 \times \mathrm{I}_{4}-80.379 \times \mathrm{I}_{1}=0$ ). Additionally, all the positions of the gaussians in the fitting curve were fixed to the values:

$\delta_{\mathrm{i}}=\mathrm{C}-0.0, \mathrm{i}=\mathrm{n}+1 ; \mathrm{C}-1.453, \mathrm{i}=\mathrm{n}+2$; and $\mathrm{C}-3.968, \mathrm{i}=\mathrm{n}+3$, for $\mathrm{n}=0,3$, and 6 .

$\mathrm{C}$ is the "best center" of the fitting curve. $\mathrm{C}$ had to be found by iteration starting from the center of the highest-intensity gaussian in the three gaussian fitting curve for the measured hexane spectrum after DOPPLER treatment (e.g., $\delta_{2}=\delta_{5}=\delta_{8}=509.551 \mathrm{keV}$ for $\mathrm{C}=511.004 \mathrm{keV}$ ). About four iteration steps were normally enough to get a good $C$-value, as judged from an acceptable variance of the fit and residual plot. GAUSSFIT does not support the use of relations between positions of the fitting gaussians, i.e., the positions can only be used as fitting parameters if they are independent. Hence, this was the only practical method to use fixed positions in agreement with the shifts in positions measured for the $\mathrm{DB}$ resolution curve in the fit. 
All the measured spectra were fitted between $\pm 3.83 \mathrm{keV}$, because the AC-spectrum for hexane was measured and fitted for angles between roughly $\pm 15 \mathrm{mrad}(3.83 \mathrm{keV})$.

The result of the fit of the pure hexane spectrum was that the variance of the fit and the residual plot were acceptable. Furthermore, one extra, free gaussian in the fitting resulted in a negative intensity of about $1 \%$, a fwhm about $3.5 \mathrm{keV}$, and, of course, a slightly better variance of the fit and residual plot. A similar fit of the measured benzene DB-spectrum in terms of the three gaussian fitting curve of the AC-spectrum for benzene gave approximately the same result. The slightly better fit if one extra gaussian was added is probably due to a difference in the subtraction of the background between the AC- and DB-cases. Hence, the main results were that the measured $\mathrm{DB}$ - and $\mathrm{AC}$-spectra for hexane and benzene agreed well within the uncertainty of the measurements and analyses.

The DB-spectra for the $\mathrm{SF}_{6}$ hexane solutions were also fitted by the expected, pure-hexane DBcurve. However, $\mathrm{SF}_{6}$ inhibits the ortho-Ps yield, $\mathrm{I}_{3}$, in hexane from $42.5 \%$ in pure hexane to $12.6 \%$ at $0.8 \mathrm{M}$, as derived from three term fits of the LT-spectra [5] (see below). Hence, we calculated the expected para-Ps yield, $I_{1}$, as $I_{1}=(3 \times 16.66 / 42.5)\left(I_{3} / 3\right)$, where $I_{3}$ was taken from the solid line through the points of fig. 2 in [5]. The first parenthesis accounts for the fact that $I_{1}$ is always expected to be larger than $\mathrm{I}_{3} / 3$ for such hexane solutions, see table 1 . The shape of the narrow peak in the fitting curves was then fixed to be identical to the shape of the narrow peak in the expected DB-curve given by the first three gaussians ( $i=1-3)$ in the curve DB hex.cal. in table 1. Fixed fwhm's and two linear combination of intensities equal to zero were used for that purpose. Its relative intensity curve was fixed to be equal to the calculated $I_{1}$ by use of another linear combination of intensities equal zero. The shape of the curve given by the other six gaussians in the expected DB-curve was fixed as in the fits discussed above. All the $\mathrm{SF}_{6}$ hexane solution spectra were fitted in that way.

At $0.001 \mathrm{M}$ and $0.002 \mathrm{MSF}_{6}$ the results were very similar to that obtained for the pure hexane DB-spectrum. Above $0.002 \mathrm{M}$ the "variance of fit" in the fits in terms of only the expected hexane $\mathrm{DB}$-spectrum increased strongly for increasing $\mathrm{SF}_{6}$ concentrations. We then fitted the spectra by use of the pure-hexane curve plus one or more extra gaussians with free parameters. The results showed that only one extra gaussian was necessary to give acceptable fits. Furthermore, at $\mathrm{SF}_{6}$ concentration above $0.02 \mathrm{M}$ the fwhm of this extra gaussian was very close to $3.45 \mathrm{keV}$. Hence, in the final analysis of all $\mathrm{SF}_{6}$ hexane solution curves the curves were fitted by the nine gaussians giving the shape of the pure hexane curve, corrected for Ps inhibition as discussed above, plus one gaussian of fwhm $=3.45 \mathrm{keV}$ and position fixed to the derived value of $\mathrm{C}$. The relative intensity of this gaussian is plotted as a function of the $\mathrm{SF}_{6}$ concentration in fig. 1 . For example, $0.005,0.005$, and $0.01 \mathrm{M} \mathrm{SF}_{6}$ gave $1.20,1.72$, and $5.53 \%$, respectively. We could not determine the shapes of the three components of the total broad AC-component. Hence, we know only that the total broad component of each spectrum, described by the determined sum of the $3.45 \mathrm{keV}$ gaussian and the broad component of the DB-resolution -broadened hexane curve, is the sum of the free positron, the trapped positron on $\mathrm{SF}_{6}$, and the pick-off components, in general.

In table 2 the H-parameter and the fwhm of the broad component of selected curves are shown. They were calculated by use of only the broad gaussians of the fitting curves, i.e., the narrow gaussians describing the para-Ps component were not included. Furthermore, they were calculated as if they were fitting curves of AC-spectra measured with a resolution fwhm $=1.15$ mrad, to be able to compare them directly with the previously determined [11] parameters.

We fitted also the $0.05 \mathrm{M} \mathrm{SF}_{6}$ spectrum without subtraction of $10 \%$ of the Kapton spectrum to test the importance of the source correction. The final analysis fit mentioned above resulted in an increase of the variance of the fit by a factor of 2.26 , a clear evidence of a too narrow fitting 
Table 2. H-parameter $(\mathrm{H})$ and full width at half maximum (fwhm) of the broad component of selected AC-curves, as if they were measured with a resolution $\mathrm{fwhm}=1.15 \mathrm{mrad}$.

\begin{tabular}{lrrr} 
Spectrum & $\begin{array}{c}\mathrm{H} \\
\left(\mathrm{mrad}^{-1}\right)\end{array}$ & $\begin{array}{c}\text { fwhm } \\
(\mathrm{mrad})\end{array}$ & ref. \\
\hline $1 \mathrm{M} \mathrm{SF}_{6} /$ hexane & 0.0972 & 9.411 & this work \\
$\mathrm{SF}_{6}$ liquid $-44 \mathrm{C}$ & 0.0830 & 11.004 & this work \\
$\mathrm{SF}_{6}$ solid $-88 \mathrm{C}$ & 0.0765 & 12.282 & this work \\
pure hexane & 0.1075 & 8.685 & {$[11]$} \\
pure $\mathrm{C}_{7} \mathrm{~F}_{14}$ & 0.0837 & 10.960 & {$[11]$} \\
pure $\mathrm{C}_{6} \mathrm{~F}_{6}$ & 0.0858 & 10.725 & {$[11]$} \\
$1.11 \mathrm{M} \mathrm{C}_{6} \mathrm{~F}_{14} /$ hex. & 0.0977 & 9.440 & {$[11]$} \\
$1.01 \mathrm{M} \mathrm{C}_{6} \mathrm{~F}_{14} /$ isooc. & 0.0977 & 9.432 & {$[11]$} \\
\hline
\end{tabular}

curve in the residual plot, and a change in the extra gaussian intensity from $12.38 \%$ to $15.90 \%$. Hence, the source correction is, certainly, necessary.

We also measured high-quality LT-spectra for the same samples and source as used in the DBmeasurement. Fits in terms of three exponentially decaying functions gave long-lifetime intensities very close to those reported in fig. 2 of [5]. However, four and/or constrained-five term fits showed the existence of the so-called "fourth" lifetime component, which is caused by secondary reaction of Ps with positron-spur reactants, in particular the positive ions. In pure hexane at least $6 \%$ of the total intensity of the LT-spectra is the fourth component, which is a distribution of exponentially decaying terms. On addition of electron acceptors the excesselectron positive-ion recombination time is strongly increased from about 10 ps to several ns because of trapping of the electrons on the acceptors. This results in an increased probability of the Ps secondary reaction with the positive ions. For example, the fourth component intensity is about $4.7 \%$, compared to a long-lived ortho-Ps yield of $11.2 \%$, at $0.5 \mathrm{M} \mathrm{SF}_{6}$ in hexane. The LTresults will be discussed in detail in another paper [19].

\section{Discussion}

The agreement within the uncertainty of the measurements and analysis between the DB-curves for hexane, benzene, and $0.001 \mathrm{M}$ and $0.002 \mathrm{M} \mathrm{SF}_{6}$ in hexane, as calculated from the three gaussian fits of the AC-spectra for hexane, benzene, hexane, and hexane, respectively, and the measured curves after DOPPLER treatment is an important result. It shows that the AC- and DBmeasurements determine identical properties of the annihilation processes apart from the resolution broadenings of the set-ups. This is a well-known result of the theory of positron annihilation [4]. However, a detailed experimental test of this result with an accuracy approaching that reported in this work has not been reported previously, as far as we know.

As discussed in the introduction we expected to measure a broadening of the DB-spectra due to the reaction of the free positron with the $\mathrm{SF}_{6}$ molecules resulting in a preferential annihilation with the electrons in fluorine. Clearly, fig. 1 shows the expected result. In [11] the similar reactions of the positron with perfluorohexane $\left(\mathrm{C}_{6} \mathrm{~F}_{14}\right)$ in hexane and isooctane were discussed in details. In particular, it was argued that a reaction of $P_{s}$ with $C_{6} F_{14}$ did not occur since the ortho-Ps lifetime is not decreased with increasing $\mathrm{C}_{6} \mathrm{~F}_{14}$ concentration, as has always been the case in previously investigated Ps reactions. A very detailed discussion in [11] showed that only a positron reaction with $\mathrm{C}_{6} \mathrm{~F}_{14}$ could explain the measured results. The same arguments apply 


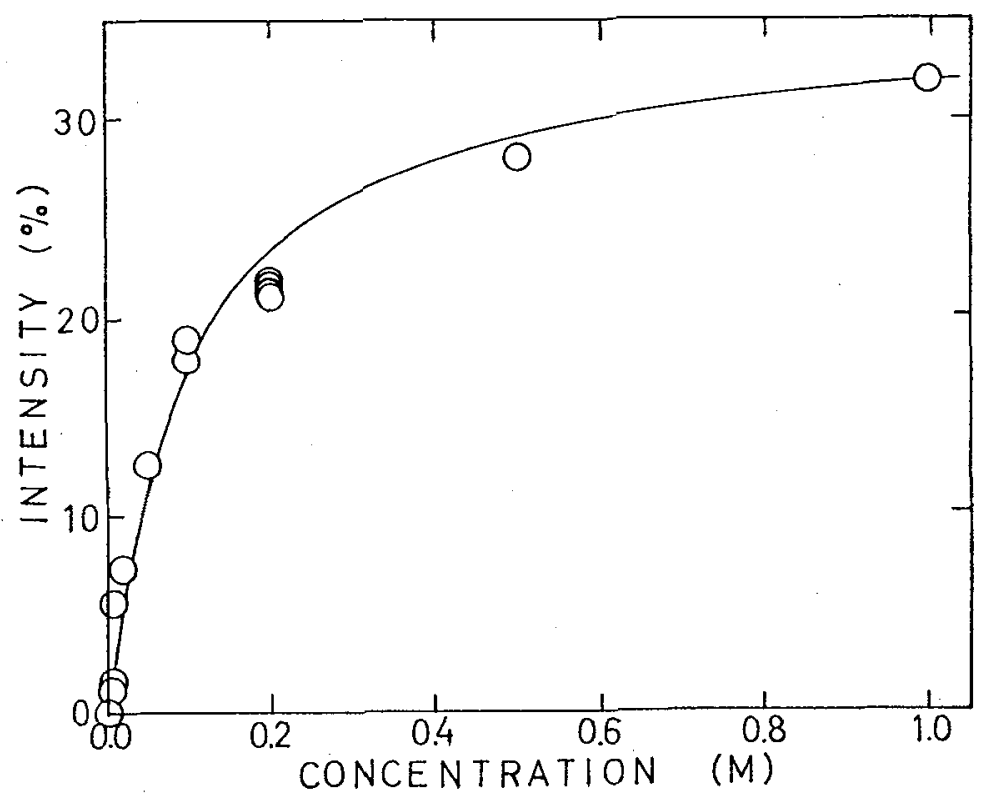

Fig. 1. The relative intensity of the broad gaussian ( $\mathrm{fwhm}=3.45 \mathrm{keV}$ ) in the final fits of the DB-spectra versus the concentration of $\mathrm{SF}_{6}$ in hexane.

for the $\mathrm{SF}_{6} /$ hexane case. Hence, we shall not repeat the detailed discussion here.

The positron reaction with $\mathrm{SF}_{6}$ occurs in competition with the annihilation. Hence, the rate equation is:

$$
\mathrm{dc}_{+} / \mathrm{dt}=-\lambda_{\mathrm{f}} \mathrm{c}_{+}-\mathrm{k}_{\mathrm{f}} \mathrm{c}_{+} \mathrm{c}_{\mathbf{a}}
$$

where $\lambda_{f}$ is the positron annihilation rate in the solution $\left(\lambda_{f}=1 /(430 \mathrm{ps})=2.3 \times 10^{9} \mathrm{~s}^{-1}\right), \mathrm{c}_{+}\left(\mathrm{c}_{\mathrm{a}}\right)$ is the concentration of the positrons $\left(\mathrm{SF}_{6}\right)$, and $\mathrm{k}_{\mathrm{f}}$ is the rate constant. Hence, the fraction of positrons annihilating in the bound state in $\mathrm{SF}_{6}, \mathrm{x}$, is:

$$
x=k_{\mathrm{f}} c_{\mathrm{a}} /\left(\lambda_{\mathrm{f}}+\mathrm{k}_{\mathrm{f}} \mathrm{c}_{\mathrm{a}}\right)
$$

This expression is only approximately correct. We have disregarded the influence of the other reactants in the positron spur on the reaction. Furthermore, we have neglected that the $\mathrm{SF}_{6}$ concentration is so high that the time dependence of $k_{f}$ and the initial encounter pair effect might play a significant role at the highest $\mathrm{SF}_{6}$ concentrations [15].

As seen in fig. 1 , the broad-gaussian intensity saturates at higher $\mathrm{SF}_{6}$ concentration. $\mathrm{A}$ very similar effect was obtained on adding $\mathrm{C}_{6} \mathrm{~F}_{14}$ to hexane, and the $\mathrm{H}$-parameters at $1 \mathrm{M} \mathrm{C}_{6} \mathrm{~F}_{14}$ and $1 \mathrm{M} \mathrm{SF}_{6}$ in hexane (table 2) are very close. Hence, in a good approximation we can assume that $35 \%$ of this intensity corresponds to $100 \%$ positron trapping on $\mathrm{SF}_{6}(\mathrm{x}=1.0)$. Furthermore, from (9) we get:

$$
x /(1-x)=\left(k_{f} / \lambda_{f}\right) c_{a}
$$


which can be used to determine $k_{f}$ from the slope of a line through the points of a plot of $x /(1$ $x$ ) versus $c_{a}$. We obtained $k_{f}=2.3 \times 10^{10} \mathrm{M}^{-1} s^{-1}$, which corresponds to the solid line in fig. 1 . Taking into account the approximations used the uncertainty is about $\pm 0.5 \times 10^{10} \mathrm{M}^{-1} \mathrm{~s}^{-1}$.

The derived $\mathrm{k}_{\mathrm{f}}$-value is somewhat lower than could be expected. We anticipate an about ten times higher rate if the reaction is diffusion controlled according to the Smoluchowski equation, and on taking into account that the positron diffusion constant in hexane is much higher than that of heavy reactants, because the anti-inhibition effect is found for hexane. The only other known rate constants for positron reactions in nonpolar liquids are those of the reaction with $\mathrm{C}_{6} \mathrm{~F}_{14}$ in hexane and isooctane, for which $3 \times 10^{10} \mathrm{M}^{-1} \mathrm{~s}^{-1}$ was determined for both solvents [11].

However, the most important result is the fact that a positron reaction with $\mathrm{SF}_{6}$ actually occurs. This confirms the prediction of the improved spur model of Ps formation [9] that a Ps inhibition. takes place only if both the positron and the electron are trapped on those molecules which forms weakly bound, stable anions on electron reaction with the molecules. $\mathrm{C}_{6} \mathrm{~F}_{14}$ and $\mathrm{SF}_{6}$ inhibit Ps formation, while $\mathrm{C}_{6} \mathrm{~F}_{6}$ gives anti-inhibition, in hexane. This is in agreement with the experimental fact that the positron reacts with $\mathrm{C}_{6} \mathrm{~F}_{14}$ and $\mathrm{SF}_{6}$, but not with $\mathrm{C}_{6} \mathrm{~F}_{6}$, in hexane. Hence, the new, experimental results on the positron reactions speak strongly in favor of the improved spur model.

In addition, the obtained results give information on a positron reaction and the formed positron $\mathrm{SF}_{6}$ bound state. Compared with the situation for the other two light particles, the excess electron and Ps, very little is known on the positron reactions in liquids. The sparse results available today show that positron reactions ought to be studied in much more detail by use of DB- and AC-measurements in the future.

The H-parameter and fwhm of the broad components in table 2 show that the shape of the $1 \mathrm{M}$ $\mathrm{SF}_{6}$ / hexane broad component is very close to that of the $1.11 \mathrm{M} \mathrm{C}_{6} \mathrm{~F}_{14} /$ hexane and $1.01 \mathrm{M} \mathrm{C}_{6} \mathrm{~F}_{14} /-$ isooctane solutions, and very different from those of pure $\mathrm{SF}_{6}$, hexane, $\mathrm{C}_{6} \mathrm{~F}_{14}$, and $\mathrm{C}_{6} \mathrm{~F}_{6}$. Because of the high vapor pressure above $\mathrm{SF}_{6}$ even at $-44 \mathrm{C}$ the measured "SF${ }_{6}$ liquid $-44 \mathrm{C}^{\text {" }} \mathrm{AC}$-curve was somewhat asymmetric. In spite of this, the parameters of this curve given in table 1 were fairly well determined. As discussed in [11], the Ps bubble state has a lower energy if the bubble surface consists of hexane molecules in $\mathrm{C}_{6} \mathrm{~F}_{14}$ hexane mixtures. Only at very high $\mathrm{C}_{6} \mathrm{~F}_{14}$ concentration a very broad Ps pick-off annihilation component of the AC spectrum characteristic of annihilation with electrons in fluorine is measured and hence, $\mathrm{C}_{6} \mathrm{~F}_{14}$ is part of the bubble surface. It would be very interesting, indeed, to investigate the $\mathrm{SF}_{6}$ hexane mixtures to see whether a similar strong broadening of the pick-off $\mathrm{AC}$ - and/or DB-components is measured only at very high $\mathrm{SF}_{6}$ concentration in the $\mathrm{SF}_{6}$ case, too. The $\mathrm{SF}_{6}$ / hexane mixtures show a miscibility gap though [5]. Clearly, the parameters shown in table 2 indicate that only about $40 \%$ of the total change of the broad component from hexane to liquid $\mathrm{SF}_{6}$ has occurred at $1 \mathrm{M} \mathrm{SF}_{6}$. This is almost identical to what was found for the $\mathrm{C}_{6} \mathrm{~F}_{14}$ solutions.

The AC-curve for solid $\mathrm{SF}_{6}$ (table 1) shows that no Ps is formed in solid $\mathrm{SF}_{6}$. This could probably be expected from Ps yield results for several other electron scavengers. For example, Ps is not formed in solid anthracene although a fairly normal Ps yield is found for liquid anthracene. The discussion in [20] concerns probably also solid $\mathrm{SF}_{6}$. It was also expected that the broad component would be broader in solid than in liquid $\mathrm{SF}_{6}$, as measured.

Finally, let us discuss the new analysis of the DB-spectra described in this work. The greatest problems in the interpretation of DB-spectra compared to that of the AC-spectra are: A) the poor resolution of the DB-setup $(-1.3 \mathrm{keV})$ compared with that of the linear-slit AC-apparatus $(\sim 0.3$ $\mathrm{keV}), \mathrm{B})$ the asymmetric resolution of the DB-setup compared with a mainly symmetric 
resolution of the $\mathrm{AC}$-apparatus, and $\mathrm{C}$ ) the fact that annihilation in the positron source is included in the normally measured (but not in slow-positron irradiated) DB-spectra, but not in the AC-spectra. In most papers the DB-spectra have been used in a semi-quantitative way. Only the $\mathrm{H}$ - and $\mathrm{W}$-parameters are normally reported, and the energy windows used in the calculation of the parameters are not given. Furthermore, background subtraction is not used or reported, and the shape of the resolution curve is not reported either. Hence, a quantitative comparison of the DB-spectra with DB-spectra and/or AC-spectra measured by other groups is impossible. Some groups deconvolute the DB-spectra for the effects of the DB-resolution. However, deconvolution of DB-spectra is a notoriously difficult task, as is well-known. Incorrect interpretations may easily occur as a result of such deconvolution.

The treatment of the DB-spectra described in sect. 3 and the use of the AC spectra in the analyses constitute a new, very useful analysis method. To our knowledge a similarly detailed comparison of the AC- and DB-spectra has not been performed before. As the resolution of the AC-setup is much smaller than that of the DB-setup, the deconvolution of the AC-spectra for the effect of the resolution introduces only very small errors in the deconvoluted AC-curves compared to a deconvolution of the DB-curves. Furthermore, the convolution of the true DB- and AC-curve with the resolution of the DB-setup and the comparison of the expected DB-curve with the measured DB-curve result in fewer and smaller errors than a deconvolution of the DBspectra followed by a comparison of the resulting curve with other deconvoluted DB- or ACspectra. Clearly, the program GAUSSFIT is an important tool in our analyses. A comparison of the DB- and AC-data for annihilation in a phenyl ether by use of a program, PAACFIT (ACARFIT) [16,17], which can only fit the spectra in terms of symmetric sums of gaussians, gave fairly unsatisfactory results [21].

In particular, the new analyses by use of the AC-spectra will probably allow a better than usual interpretation of the DB-spectra in slow-positron research, where LT-spectra in case of Ps formation cannot normally (see [22] though) be measured, and where important information can, therefore, easily be overlooked.

\section{Conclusion}

A new method to compare AC-spectra with DB-spectra has been used to show that the AC-and DB-measurements determine the same properties of the positron annihilation in hexane and benzene. The new method of analysis was based on the application of a new computer program, GAUSSFTT. The strong broadening of the DB-spectra at low $\mathrm{SF}_{6}$ concentration indicates that the positron reacts with $\mathrm{SF}_{6}$ and annihilates from the formed bound state. This result confirms a prediction based on the improved spur model of Ps formation, namely that the inhibition of Ps formation by a molecule forming weakly bound, stable anions on the reaction with electrons is only found if the positron is also trapped on the molecules.

\section{Acknowledgements}

The authors acknowledge the development of the GAUSSFIT program by P. Kirkegaard and the testing of the program by N. J. Pedersen. They also wish to thank Dr. Y. Ito for collaboration on the measurements. 


\section{References}

[1] L. Dorikens-Vanpraet, M. Dorikens and D. Segers, eds., Positron Annihilation, Proceedings of the Eighth Conference of Positron Annihilation, Gent, Belgium, 1988 (World Scientific, Singapore).

[2] Zs. Kajcsos and Cs. Szeles, eds., Positron Annihilation, Proceedings of the Ninth Conference of Positron Annihilation, Szombathely, Hungary, 1991, Mater. Sci. Forum 105-110 (1992) 1-2070.

[3] D. M. Schrader and Y. C. Jean, eds., Positron and Positronium Chemistry (Elsevier, Amsterdam, 1988).

[4] O. E. Mogensen, Positron Annihilation in Chemical Physics (Springer, Heidelberg, to be published).

[5] B. Levay and O. E. Mogensen, Chem. Phys. $\underline{53}$ (1980) 131.

[6] O. E. Mogensen, Chem. Phys. Lett. 65 (1979) 511.

[7] P. Jansen and O. E. Mogensen, Chem. Phys. 25 (1977) 75.

[8] G. Wikander, Chem. Phys. Lett. 80 (1981) 361.

[9] G. Wikander, O. E. Mogensen and N. J. Pedersen, Chem. Phys. 77 (1983) 159.

[10] O. E. Mogensen and F. M. Jacobsen, Chem. Phys. 73 (1982) 223.

[11] O. E. Mogensen, to be published.

[12] J. W. T. Spinks and R. J. Wood, An Introduction to Radiation Chemistry (J. Wiley, New York, 1990).

[13] P. Kirkegaard, N. J. Pedersen and O. E. Mogensen, unpublished.

[14] T. Hirade and O. E. Mogensen, Chem. Phys. 170 (1993) 249.

[15] O. E. Mogensen and N. J. Pedersen, Radiat. Phys. Chem. 28 (1986) 33.

[16] P. Kirkegaard, M. Eldrup, O. E. Mogensen and N. J. Pedersen, Comp. Phys. Comm. 23 (1981) 307.

[17] P. Kirkegaard, N. J. Pedersen and M. Eldrup, PATFIT-88, Risø-M-2740 (1989).

[18] J. T. Grissom and D. R. Koehler: Am. J. Phys. $\underline{35}$ (1967) 753.

[19] T. Hirade and O. E. Mogensen, to be published.

[20] O. E. Mogensen, in Positron Annihilation Studies of Fluids, edited by S. Sharma (World Scientific, Singapore, 1988) p. 418.

[21] O. E. Mogensen, N. J. Pedersen, B. D. Malhotra and R. A. Pethrick, Chem. Phys. $\underline{87}$ (1984) 139.

[22] N. Hayashi, R. Suzuki, M. Hasegawa, N. Kobayashi, S. Tanigawa and T. Mikado, Phys. Rev. Lett. 70 (1993) 45. 